\title{
A HISTÓRIA DA OFTALMOSCOPIA NA CIÊNCIA MÉDICA E VETERINÁRIA
}

\author{
(The history of ophthalmoscopy in medical and veterinary science) \\ Henrique de Moura Freitas ${ }^{1}$, Thiago Alegre Coelho Ferreira, Fabiano Montiani-Ferreira. \\ Universidade Federal do Paraná, Curitiba, PR, Brasil.
}

1'Autor para correspondência: henrique.mouravet@gmail.com

RESUMO - O olho tem sido um antigo objeto de curiosidade e estudos, na Babilônia já havia relatos de primitivas investigações sobre a natureza do órgão da visão, assim como no antigo Egito e Grécia. O principal objetivo do presente trabalho foi fazer uma revisão histórica do desenvolvimento do equipamento que possibilitou explorar a mística do que estaria atrás da pupila e que moveu diversos pesquisadores a estudarem as estruturas internas oculares possibilitando a descoberta de importantes condições oftálmicas em seres humanos e animais, demonstrando a importância dessa ferramenta para a medicina e medicina veterinária. O oftalmoscópio foi desenvolvido pelo físico alemão Hermann von Helmholtz, em 1851, a partir de então passou por diversas adaptações que possibilitaram a exploração de um mundo até então desconhecido: o fundo ocular. Com o crescimento das especialidades na medicina veterinária, ferramentas como 0 oftalmoscópio se incluem entre os principais utensílios do oftalmologista veterinário, mas ainda são pouco conhecidos pelos clínicos tradicionais.

Palavras-chave: oftalmologia; oftalmoscópio; animais; medicina veterinária.

ABSTRACT - The eye has been an ancient object of curiosity and studies, in Babylon there were already reports of primitive investigations into the nature of the organ of sight, just as in ancient Egypt and Greece. The main objective of the present work was to make a historical review of the development of the equipment that made it possible to explore the mystique of what would be behind the pupil and that moved several researchers to study the internal ocular structures making possible the discovery of important ophthalmic conditions in humans and animals, demonstrating the importance of this tool for medicine and veterinary medicine. The ophthalmoscope was developed by the German physicist Hermann von Helmholtz in 1851 , from then on it underwent several adaptations that allowed the exploration of a world previously unknown: the ocular fundus. With the growth of specialties in veterinary medicine, tools like the ophthalmoscope are among the top tools of the veterinary ophthalmologist, but are still little known by traditional clinicians.

Keywords - ophthalmology; ophthalmoscope; animals; veterinary medicine. 


\section{INTRODUÇÃO}

A retina é a única porção do sistema nervoso central visível do exterior. Da mesma forma, o fundo ocular é a única região anatômica na qual a vasculatura pode ser visualizada sem a necessidade de um procedimento cirúrgico. Além da oftalmologia médica e veterinária, muito do que é diagnosticado e tratado na medicina interna tem relação vascular e, portanto, a visualização do fundo de olho é uma ótima maneira de se obter informações sobre a vascularização geral do paciente e alertar sobre sinais de doenças sistêmicas (SMITH, 2014). Além disso, o exame fundoscópico pode elucidar processos patológicos mais críticos, com exemplos abundantes que incluem 0 reconhecimento de endocardite, candidemia disseminada, diabetes, hipertensão e acidente vascular cerebral (LOUGHREY et al., 2014; IMONIKHE et al., 2016; CHUNG et al., 2017; MELLINGHOFF et al., 2018; SPRÖDHUBER et al., 2018) e doenças que podem levar à cegueira (CARR, 1965, 1974).

A visualização da retina mostrase então de suma importância para a clínica médica e a invenção do oftalmoscópio por Hermann von Helmholtz possibilitou grandes descobertas e a investigação de diferentes retinopatias. $O$ presente trabalho tem o objetivo de trazer um breve histórico a respeito da invenção do oftalmoscópio e uma revisão das suas melhorias ao longo do tempo por meio de uma revisão de literatura, demostrando a sua importância e suas aplicações como ferramenta clínica para a medicina e veterinária.

\section{DESENVOLVIMENTO}

Considerações históricas sobre a oftalmologia

Em 2250 a.C, na Babilônia, foi instituído o Código de Hamurabi, tal conjunto de leis já indicava o uso de técnicas que demonstravam conhecimentos de oftalmologia. O lema "olho por olho e dente por dente" também se aplicava aos médicos da época que eram chamados para avaliar afecções odontológicas como a presença de abcessos dentários que estavam pressionando e comprometendo o olho. Caso houvesse alguma complicação e o paciente viesse a perder o seu olho, o médico também deveria ter 0 seu olho removido, segundo tal código naquela época (WHEELER, 1946).

Por volta de 1525 a.C., o papiro de Ebers foi escrito. Tal documento encontrado pelo monge alemão George Ebers, em 1873, demonstrava o uso de práticas médicas como o uso terapêutico de ervas como alho-poró e papoula para tratamento de afecções oftálmicas. O documento era composto de 110 colunas, sendo que 8 delas eram dedicadas à procedimentos oftálmicos. Os egípcios da época também utilizavam um amuleto denominado "Olho de Hórus", que tinha o objetivo de espantar o mal agouro e facilitar a caminhada para a vida após a morte (ANDERSEN, 1997), esse símbolo inclusive é utilizado até hoje pelo ACVO (Colégio Americano de Oftalmologistas Veterinários).

Em meados de 800 a.C. na Índia, Sushruta, tido como o pai da cirurgia e oftalmologia indiana, surge com suas pesquisas para desconstruir rituais mágicos que até então eram tidos como milagrosos na Índia védica. Sushruta utilizava misturas que envolviam mais de 760 plantas, leite e carne de diversas espécies de animais para seus tratamentos. Descreveu 76 doenças oculares, cinquenta e uma delas tratáveis cirurgicamente, as quais chamava de Nayana-Budbada, e as agrupou de acordo com a fisiopatologia e as estruturas anatômicas envolvidas. Também inventou técnicas de antissepsia baseando-se em fumigar a 
sala com mostarda branca, guggul, dentre outras ervas, além de ser tido por muitos como o primeiro médico a realizar facectomia extra-capsular (KANSUPADA; SASSANI,1997).

Na Grécia Antiga, Hipócrates, em 460 a.C., considerado o pai da medicina, introduziu a ciência da observação médica e o pensamento indutivo. Hipócrates acreditava na existência de 4 humores cardiais (sangue, fleuma, bile amarela e bile negra), esses causariam as doenças de acordo com o pensamento da época. Técnicas de venossecção e trepanação eram utilizadas em enfermidades de evolução aguda e leite materno, cobre, ferro eram utilizados em afecções de caráter crônico (WHEELER, 1946).

A escola de Alexandria também foi responsável por introduzir alguns conhecimentos à oftalmologia como a utilização de medicamentos aplicados topicamente na superfície ocular e a descrição da catarata e da anatomia do olho, por estudiosos como Aulo Cornélio Celso, Caio Plínio Segundo e Cláudio Galeno, nos dois primeiros séculos do primeiro milênio (WHEELER, 1946).

Por séculos tentou-se entender por que a pupila é escura e por que os olhos de alguns animais refletiam luz. Jean Méry, em 1704, fez um experimento em que ele submergiu um gato e observou pela primeira vez os vasos da retina por meio da dilatação da pupila do animal causada pela hipóxia (ÁVILA, 2003).

Todavia, o estudo do fundo ocular revolucionou-se após a descoberta do oftalmoscópio em 1851, por Hermann von Helmholtz, instituindo uma técnica denominada (HELMHOLTZ, 1851).

A invenção e o desenvolvimento dos diferentes tipos de oftalmoscópio

Hermann von Helmholtz, um físico alemão, apresentou em 1851, na Berlim Physical Society seu oftalmoscópio na sua obra Augenspiegel, esse consistia em um conjunto de placas de vidro superpostas que deveriam ser iluminadas com luz externa, assim foi criado o primeiro oftalmoscópio direto, com a formação de uma imagem ampliada, virtual e normal e um pequeno campo de visão. Esse aparelho também dispunha de lentes côncavas soltas para corrigir a ametropia do paciente e do examinador, demonstrando que um oftalmoscópio deve ter uma fonte de iluminação, uma superfície refletora e um meio de correção do foco do fundo ocular (SHERMAN, 1989), tal instrumento possibilitou a identificação detalhada do fundo ocular (FRIEDEXWALD, 1902).

Anos antes, em 1847, Charles Babbage, o inventor da calculadora, inventou um instrumento que fazia a mesma função do oftalmoscópio de von Helmholtz, no entanto, seu equipamento não foi aceito quando foi apresentado pela primeira vez a um oftalmologista da época chamado Wharton-Jones, muito provavelmente porque Wharton-Jones era míope e não conseguiu utilizar 0 aparelho, assim o equipamento não foi patenteado e von Helmholtz foi intitulado como 0 inventor do oftalmoscópio (KEELER, 1997).

Inicialmente a fonte de iluminação era a vela, no entanto, a luz proveniente da mesma era sempre muito fraca, assim, logo utilizou-se lamparinas a óleo e a gás para melhorar a qualidade da iluminação, até que em 1879 Thomas Edson inventou a lâmpada elétrica que foi implementada pela primeira vez por William Dennett em um oftalmoscópio, melhorando significativamente a qualidade da iluminação, logo Thomas Reid e Henry Juler também utilizaram essa nova tecnologia para a oftalmoscopia em 1886 (KEELER, 2002).

Após a invenção de von Helmholtz, Epkens de Amsterdam em 1851 implementou uma abertura circular 
entre as placas de vidro para facilitar a visualização pelo observador, tão logo, Egbert Rekoss, em 1852, na Alemanha, inventou os discos de Rekoss, onde foi possível fixar as lentes de correção, que antes eram soltas, em 2 discos e corrigir os erros de refração no processo de observação, pois as estruturas ópticas deixaram de produzir imagens convergentes. Após essa adaptação foi possível incluir lentes convexas e côncavas em discos diferentes ou até no mesmo disco melhorando a qualidade da fundoscopia (SHERMAN, 1989).

Em 1852, na Alemanha, Christian Theodor Ruete, professor da Universidade de Leipzig, inventou o oftalmoscópio indireto, ele utilizou um espelho côncavo perfurado ao meio que refletia a luz de uma vela no olho do paciente. A abertura do espelho era acoplada a um espelho convexo de 13 a 20 dioptrias, que colimava a luz que refletia do olho do paciente, formando uma imagem invertida com menor magnificação e por consequência, maior campo de visão (SIMONSZ, 2004).

Em 1853, Adolf Coccius, também de Leipzig, Alemanha, adaptou um espelho plano quadrado a uma lente biconvexa que poderia direcionar a luz até a abertura central do espelho, por meio de um cabo manual, esse espelho mais tarde foi trocado por um côncavo melhorando o poder de dioptrias do oftalmoscópio (KEELER, 2002).

Ulrich, da Alemanha, no mesmo ano, foi o primeiro a adaptar um sistema de tubos ao oftalmoscópio, onde eram acoplados lentes e espelhos em um tubo e em um segundo tubo uma fonte de luz dando origem a um novo oftalmoscópio indireto (SHERMAN, 1989).

Eduard Jaeger, em 1854, na Áustria, foi o primeiro a utilizar espelhos intercambiáveis, com o sistema de placas de vidro de von Helmholtz adaptado ao espelho côncavo de Ruete, ele costumava durante as consultas desenhar o fundo de olho de seus pacientes e foi o primeiro a publicar uma obra com os fundos de olho de pacientes saudáveis e doentes em 1855 (KEELER et al., 2013).

Em 1869, Edward Loring, foi o primeiro oftalmologista dos Estados Unidos a contribuir para o desenvolvimento de um oftalmoscópio. Ele foi responsável por três diferentes oftalmoscópios, o primeiro modelo, chamado de "Wecker", era constituído de dois discos destacáveis, um de lentes convexas e outro de côncavas. O segundo modelo tinha um disco com uma fileira de 23 lentes e o terceiro era constituído de um espelho que poderia ser inclinado atrás do oftalmoscópio mais um conjunto suplementar de lentes (SHERMAN, 1989) (Figura 1).

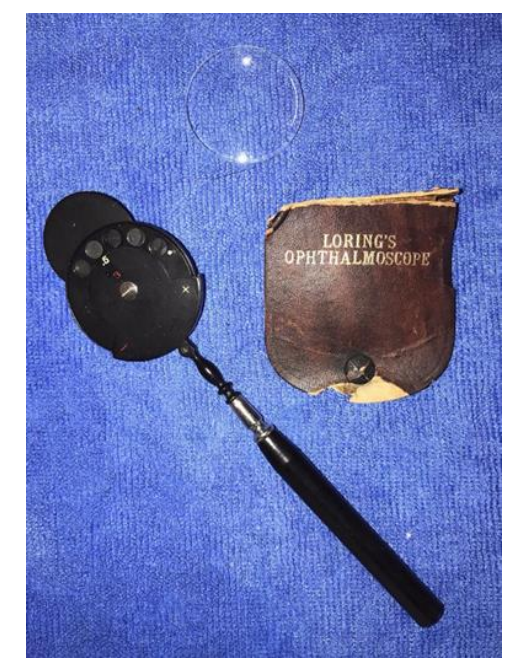

Figura 1 - Oftalmoscópio de Loring. Note a simplicidade do terceiro modelo produzido. Normalmente, vinha acompanhado de uma lente. Do outro lado do seletor de lentes do instrumento havia um espelho. Fonte: Os autores.

Um problema recorrente nos modelos de oftalmoscópio era a disposição oblíqua dos espelhos, deformando a imagem do fundo de olho, assim, em 1875, John Cooper utilizou espelhos que se inclinavam em seu eixo vertical para esquerda ou direita em direção a fonte de luz, sobre um disco 
Rekoss possibilitando o observador visualizar o fundo perpendicularmente (KEELER, 2002).

Girard Teulon inventou na França, em 1861, o primeiro oftalmoscópio binocular indireto, esse consistia de um grande espelho côncavo e dois prismas de vidro e utilizava uma lente condensadora biconvexa para realizar a oftalmoscopia indireta. Esse aparelho foi melhorado por Charles Schepens em 1946, que inventou o oftalmoscópio binocular indireto elétrico (SHERMAN, 1989).

A partir de então os oftalmoscópios ganharam importantes melhorias que facilitaram a observação das estruturas oculares, possibilitando a magnificação das imagens em até 16 vezes a partir dos oftalmoscópios coaxiais (RAMSAMY et al., 2017) (Figura 2). Outro equipamento, o oftalmoscópio Panoptic $^{\circledR}$ (Welch Allyn ${ }^{\circledR}$ ), ainda pode ser acoplado em câmeras de smartphones que possibilitam o registro fotográfico do fundo ocular com alta definição e permite um campo visual melhor do que o oftalmoscópio direto, no entanto, inferior à técnica de oftalmoscopia indireta utilizando a lente de 20D e um transiluminador ou um oftalmoscópio binocular indireto (ADAM et al., 2015; MOWAT et al., 2018) (Figura 2 e 3).

Algumas contribuições da oftalmoscopia para a clínica médica e oftalmológica

Com a invenção do oftalmoscópio foi possível identificar diversas lesões como a retinose pigmentar por Donders em 1853, doença que causa cegueira progressiva em seres humanos e pigmentação do fundo ocular. $O$ descolamento de retina e a retinose pigmentar foram observados por Coccius no mesmo ano, a escavação do nervo óptico causada pelo glaucoma foi visualizada pela primeira vez por von Graefe em 1855, que em 1860 também observou pela primeira vez 0 papiledema (PEARCE, 2009). Heyman, em 1856, descobriu a retinopatia hipertensiva e Jaeger em 1861, a atrofia do disco óptico (SHERMAN, 1989).

Em 1869, Theodor Leber descreveu a Amaurose Congênita de Leber, doença que causa grave déficit visual, nistagmo e em alguns casos pigmentação do fundo ocular, com evolução do quadro visual muito rápida. Mais tarde em 1983, Nikkoskelainen e colaboradores descreveram outras alterações do fundo de olho como microangiopatia, atenuação e aumento da tortuosidade vascular e alterações na aparência do disco óptico em pessoas diagnosticadas com essa doença (Nikkoskelainen et al., 1983).

Em 1882, Mooren descreveu pela primeira vez a retinite punctata albecens (HAMEL el al., 2014), que é um tipo agressivo de retinose pigmentar, que apresenta pontos brancos na retina.

Em 1885, Haab descreveu a degeneração macular senil, que é caracterizada como uma condição que causa déficit visual e alterações atróficas e pigmentares na região macular (GASS, 1973).

Em 1907, Ogushi caracterizou um tipo de cegueira noturna estacionária, que causava despigmentação do fundo ocular, esse tornava-se amarelo metálico com a presença da doença. $O$ aspecto do fundo ocular e os demais sinais clínicos se normalizavam após adaptação prolongada ao escuro, essa condição foi nomeada como doença de Ogushi (CARR \& GOURAS, 1965).

Em 1910, Lauber descreveu pela primeira vez um outro tipo de cegueira estacionária, semelhante a doença de Ogushi, chamada fundus albipunctatus (Carr, 1974) e diferenciou da retinite punctata albecens, que apresentava alterações do fundo de olho muito semelhantes.

A análise do fundo de olho também possibilitou 0 auxílio no diagnóstico de doenças degenerativas 
como a esclerose múltipla. Nessa doença ocorre atrofia da camada de fibras nervosas em decorrência da neurite óptica, característica que pode ser observada por meio da palidez do disco óptico (FRISEN et al.,1974).

Contribuições da oftalmoscopia para a medicina veterinária

$$
\text { Apesar de existirem }
$$

descrições de práticas de medicina primitiva datadas do Egito Antigo, o surgimento das escolas veterinárias é relativamente recente. Os primeiros centros de estudo veterinário da Europa e EUA surgiram entre os séculos XVIII e XIX (BRESSOU, 1970).

A oftalmologia comparativa surgiu nesse mesmo período tendo como foco principal os cavalos que eram os principais animais estudados pela medicina veterinária até a Primeira Guerra Mundial. Os primeiros livros de oftalmologia veterinária datam do século XIX, na França, esses foram escritos por Eugene Nicolas e Urbain Leblanc. $\mathrm{Na}$ mesma época Von Helmholtz desenvolveu o primeiro oftalmoscópio, técnica que possibilitou a visualização de estruturas oculares profundas e a identificação das principais doenças do fundo ocular em seres humanos e animais (GELATT, 2008).

Dentre as principais doenças descritas na oftalmologia veterinária estão a atrofia progressiva de retina e a anomalia ocular do Collie. Magnusson (1911) descreveu a atrofia progressiva de retina, doença semelhante a retinose pigmentar que causa degeneração da retina e cegueira em cães e gatos de raça pura (PETERSEN-JONES, 1998).

Roberts (1969) descreveu a anomalia ocular do Collie, condição hereditária que tipicamente se apresenta com hipoplasia vascular da coroide e coloboma do disco óptico. Outras lesões como o edema e o descolamento de retina e a diminuição de calibre dos vasos sanguíneos, típicas de distrofias de retina, além de alterações no fundo ocular que podem indicar até mesmo alguns tipos de neoplasias puderam ser identificadas em diversas espécies de animais por meio dessa técnica (ALINA et al., 2008). Muitas foram as contribuições da oftalmoscopia para a oftalmologia comparada, no entanto, poucas são as descrições e estudos da técnica na literatura veterinária especificamente. Talvez, por isso trata-se de uma técnica para a qual a maioria dos veterinários não se sinta familiarizado. Dentre os poucos estudos disponíveis na literatura, um realizado nos Estados Unidos da América (EUA) se destaca. Foi investigada, por meio de questionário, a familiaridade com os exames oftalmoscópicos de médicos veterinários do estado da Carolina do Norte nos EUA. A pesquisa envolvia perguntas acerca do treinamento recebido na graduação, frequência na qual realizavam o exame de fundo de olho, os métodos utilizados para dilatar a pupila e se os participantes acreditavam que necessitariam de mais treinamento. Do total de entrevistados apenas $10,2 \%$ responderam 0 questionário, totalizando 231 profissionais. A maioria dos entrevistados afirmaram que nunca realizavam o exame ou faziam de uma a duas vezes por semana ou por mês. Quanto aos métodos para a dilatação da pupila a grande maioria $(85,8 \%)$ dos entrevistados não utilizavam nenhum método medicamentoso ou não realizam o exame, sendo que destes (49,4\%) realizavam o exame em sala escura. Apenas 32 dos entrevistados (13,9\%) referiram utilizar métodos de oftalmoscopia indireta como 0 seu método principal de exame (Figura 3). A maioria dos entrevistados afirmou que não possuíam confiança completa na realização desse exame, nem foram submetidos a algum tipo de treinamento (MOWAT et al., 2018). 


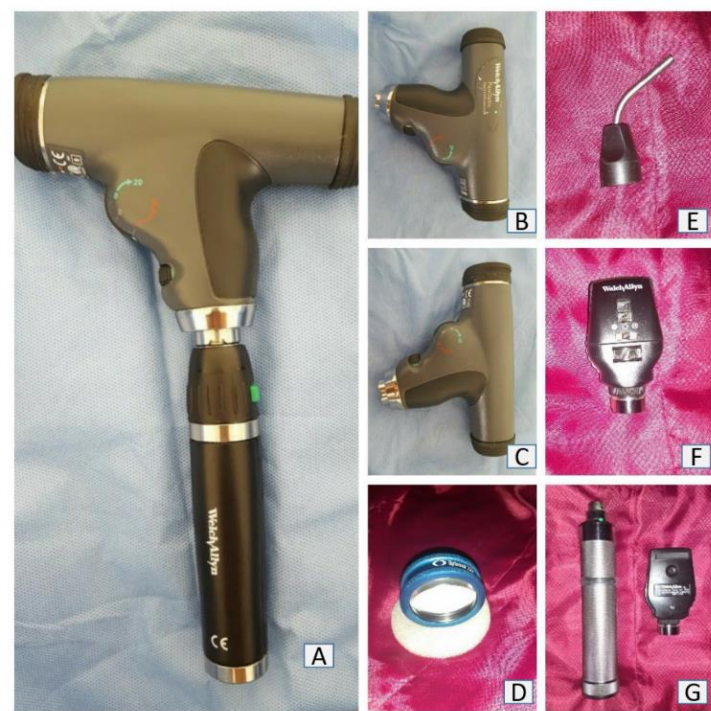

Figura 2 - (A) Oftalmoscópio ${ }^{\circledR}$ Panoptic com cabo Li-lon de 3,5V, Welch Allyn®, permite um campo de visão intermediário entre a oftalmoscopia direta e indireta. $(B, C)$ Vista lateral do Oftalmoscópio ${ }^{\circledR}$ Panoptic. (D) Lente de 20D, Optomed®, utilizada para oftalmoscopia indireta. (E) Trasiluminador Finoff, Welch Allyn®, utilizado em conjunto com a lente de 20 D. (F) Oftalmoscópio co-axial Welch Allyn®, utilizado para oftalmoscopia direta. (G) Oftalmoscópio coaxial e cabo NI-CAD de 3,5V, Welch Allyn®. Fonte: Os autores.

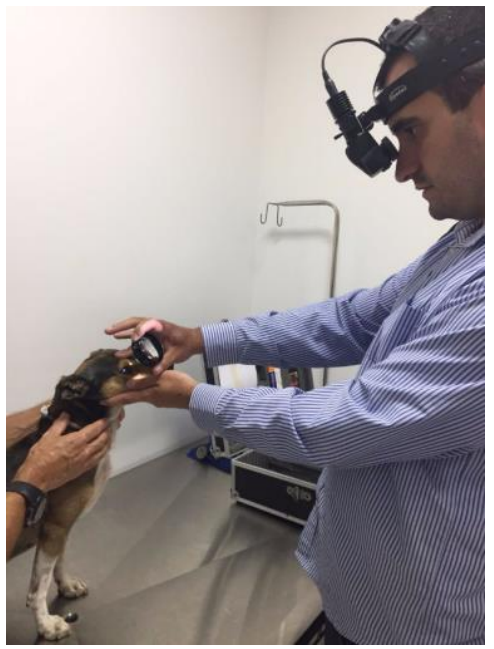

Figura 3 - Correto posicionamento de um oftalmoscópio binocular indireto e lente de 20D durante o exame de um cão. Fonte: Os autores.

\section{CONCLUSÃO E CONSIDERAÇÕES FINAIS}

O homem vem buscando compreender o funcionamento do olho desde a antiga Babilônia, passando a oftalmologia por processos evolutivos desde a imersão de um gato em um lago para nutrir a curiosidade de o que haveria atrás do buraco negro chamado pupila, até sofisticados métodos de observação do fundo ocular. A invenção do oftalmoscópio foi de fundamental importância para maior compreensão da oftalmologia geral e principalmente do funcionamento e distúrbios da retina, configura-se, portanto, um marco na história da oftalmologia o antes e o depois de von Helmholtz. Essa técnica permitiu a identificação de importantes lesões de fundo de olho e é uma das mais importantes ferramentas de diagnóstico de lesões de retina em seres humanos e animais. Com o crescimento das especialidades dentro da medicina veterinária é uma tendência a ampla inclusão desses aparelhos entre os veterinários oftalmologistas e também da clínica médica, no entanto, são necessários aprimoramentos e educação continuada após a formação dos estudantes de medicina veterinária para maior compreensão da técnica, uma vez que a maioria dos médicos veterinários não se familiarizam com ela ou não se sentem plenamente confiantes na sua utilização.

\section{REFERENCIAS}

ADAM, M. K.; BRADY, C. J., FLOWERS, A. M. et al. Quality and diagnostic utility of mydriatic smartphone photography: the Smartphone Ophthalmoscopy Reliability Trial. Ophthalmic Surgery, Lasers and Imaging Retina, v. 46, n. 6, p. 631-637, 2015.

ALINA, D.; MUSTE, A.; OANĂ, L. et al. Eye fundus examination in animals and its importance for diagnoses. Lucrări Stiinłifice Medicină Veterinară, v. 18, p. 101-130, 2008.

ANDERSEN, S. R. History of Ophthalmology. The eye and its 
diseases in Ancient Egypt. Acta Ophthalmologica Scandinavica, v. 75, p. 338-344, 1997.

ÁVILA, M. A retina no século $X X I$. Arquivos Brasileiros de Oftalmologia, v. 66, n. 5, p. 719-730, 2003.

BRESSOU, C. History of veterinary medicine. Bulletin de l'Academie veterinaire de France, v. 43, n. 9, p. 393-394, 1970.

CARR, R. E.; GOURAS, P. Oguchi's disease. Archives of Ophthalmology, v. 73 , n. 5 , p. $646-656,1965$.

CARR, R. E. Congenital stationary nightblindness. Transactions of the American Ophthalmological Society, v. 72, p. 448-87, 1974.

CHUNG, G.C.; YOON K.C.; KIM, W.H. Multiple Subretinal and Intraretinal Hemorrhages as a First Sign of Infective Endocarditis. Journal of the Korean Ophthalmological Society, v. 58, n. 12, p. 1416-1419, 2017.

FRIEDEXWALD, $\mathrm{H}$. The history of the invention and op the development of the ophthalmoscope. Transactions, v. 52, p. 89, 1902.

FRISÉN, L; HOYT, W. F. Insidious atrophy of retinal nerve fibers in multiple sclerosis: funduscopic identification in patients with and without visual complaints. Archives of Ophthalmology, v. 92, n. 2, p. 91-97, 1974.

GASS, J. D. M. Drusen and disciform macular detachment and degeneration. Archives of Ophthalmology, v. 90, n. 3, p. 206-217, 1973.

GELATT, P. K. N. Veterinary Ophthalmology: Our Past, Present and Future. Bulletin de l'Académie
Vétérinaire de France. v. I, n. 1, p. 299-306, 2008.

HAMEL, C.; DESSALCES, E.; MEUNIER, I. Retinitis Punctata Albescens. In: Inherited Chorioretinal Dystrophies. Springer, Berlin, Heidelberg, 2014. p. 135-141.

HELMHOLTZ, H. von. Beschreibung des Augenspiegels. In: Beschreibung Eines Augen-Spiegels. Springer Berlin Heidelberg. p. 28-34, 1851.

IMONIKHE, R. J.; FINER, N.; GALLAGHER, $K$. et al. Direct ophthalmoscopy should be taught to undergraduate medical students. Eye (Basingstoke), v. 30, n. 3, p. 497, 2016.

KANSUPADA, K. B.; SASSANI, J.W. Sushruta: The father of Indian surgery and ophthalmology. Documenta Ophthalmologica, v. 93, p. 159-167, 1997.

KEELER, C. R. The Ophthalmoscope in the Lifetime of Hermann von Helmholtz. Archives of Ophthalmology, v. 120, n. 2, p. 194, 2002.

KEELER, C. R. 150 years since Babbage's ophthalmoscope. Archives of ophthalmology, v. 115, n. 11, p. 1456-1457, 1997.

KEELER, C. R.; SINGH, A. D.; DUA, H. S. A master mastering the ophthalmoscope: Eduard von Jaeger. British Journal of Ophthalmology, v. 97, n. 4, p. 387-388, 2013.

LOUGHREY, P. B.; ARMSTRONG, D.; LOCKHART, C. J. Classical eye signs in bacterial endocarditis. Qjm: An International Journal of Medicine, v. 108, n. 11, p. 909-910, 2015.

MAGNUSSON, $\quad H$. Uber retinitis pigmentosa und Konsinguinitat beim 
Hunde. Arch Vergleichende Ophthal, v. 2, p. 147-149, 1911.

MELLINGHOFF, S. C.; HOENIGL, M.; KOEHLER, $P$ et al. EQUAL Candida Score: An ECMM score derived from current guidelines to measure QUAlity of Clinical Candidaemia Management. Mycoses, v. 61, n. 5, p. 326-330, 2018.

MOWAT, F. M.; ROYAL, K. D.; WESTERMEYER, $H$. D. Ophthalmoscopy skills in primary care: a cross-sectional practitioner survey. Veterinary Record, v. 32, p. 9-11, 2018.

NIKOSKELAINEN, E.; HOYT, W.F.; NUMMELIN, K. Ophthalmoscopic findings in Leber's hereditary optic neuropathy: II. The fundus findings in the affected family members. Archives of Ophthalmology, v. 101, n. 7, p. 1059-1068, 1983.

PEARCE, J. M. S. The ophthalmoscope: Helmholtz's Augenspiegel. European Neurology, v. 61, n. 4, p. 244-249, 2009.

PETERSEN-JONES, S. M. A review of research to elucidate the causes of thegeneralized progressive retinal atrophies. The Veterinary Journal, $v$. 155, n. 1, p. 5-18, 1998.

RAMSAMY, G.; ARUNAKIRINATHAN, M.; COOMBES, A. The essentials of fundoscopy. British Journal of Hospital Medicine, v. 78, n. 2, p. C28C32, 2017

SHERMAN, S. E. The history of the ophthalmoscope.

Documenta

Ophthalmologica, v. 71 , n. 2, p. 221228, 1989.

SIMONSZ, H. J. Christian Theodor Georg Ruete: the first strabismologist, coauthor of listing's law, maker of the first ophthalmotrope and inventor of indirect fundoscopy. Strabismus, v. 12, n. 1, p. 53-57, 2004.

SMITH, K. Clinical examination and diseases of the fundus in dogs. Practice, v. 36, n. 7, p. 315, 2014.

SPRÖDHUBER, A.; WOLZ, J.; BUDAI, A. The role of retinal vascular density as a screening tool for ageing and stroke. Ophthalmic Research, v. 60, n. 1, p. 18, 2018.

WHEELER, J. R. History of Ophthalmology Through the Ages. The British journal of ophthalmology, $v$. 30, n. 5, p. 264-75, 1946. 